\title{
Análise da inclinação tibial posterior como fator de risco para lesão do ligamento cruzado anterior
}

\section{Analysis of Posterior Tibial Slope as Risk Factor to Anterior Cruciate Ligament Tear}

\author{
Pedro Guilme Teixeira de Sousa Filho ${ }^{1,2} \quad$ Andre Cavalcante Marques ${ }^{10} \quad$ Leonardo Soares Pereira $^{10}$ \\ Breno Almeida Pigozzo ${ }^{10}$ Rodrigo Sattamini Pires e Albuquerque ${ }^{1 \oplus}$
}

\author{
${ }^{1}$ Centro de Cirurgia do Joelho, Instituto Nacional de Traumatologia e \\ Ortopedia (INTO), Rio de Janeiro, RJ, Brasil \\ ${ }^{2}$ Grupo GENU, Fortaleza, CE, Brasil
}

Endereço para correspondência Pedro Guilme Teixeira de Sousa Filho, MD, MSc, Instituto Nacional de Traumatologia e Ortopedia, Rio de Janeiro, RJ, Brasil (e-mail: drpedroguilme@gmail.com).

Rev Bras Ortop 2021;56(1):47-52.

\section{Resumo \\ Palavras-chave \\ - ligamento cruzado anterior \\ - ligamentos \\ - traumatismos do joelho \\ - tíbia}

\begin{abstract}
Objective The objective of the present study was to evaluate the relationship between patients with anterior cruciate ligament $(\mathrm{ACL})$ injury by indirect trauma and increased posterior tibial inclination.
\end{abstract}

recebido

08 de Março de 2019

aceito

20 de Fevereiro de 2020

Publicado online

Setembro 22, 2020
Objetivo O objetivo do presente estudo foi avaliar a relação entre pacientes com lesão do ligamento cruzado anterior (LCA) por trauma indireto e o aumento da inclinação posterior da tíbia.

Métodos Estudo retrospectivo, realizado por análise de prontuários e radiografias digitais de pacientes, presentes em banco de dados de um hospital terciário de ortopedia e traumatologia. A amostra foi composta por dois grupos, sendo o primeiro formado por pacientes com diagnóstico de lesão do LCA, por trauma indireto, e um grupo controle pareado por idade.

Resultados Cada grupo foi formado por 275 pacientes, cujas medidas de inclinação tibial posterior foram aferidas por 3 especialistas. Observou-se que o grupo dos pacientes com lesão do LCA apresentou slope tibial (em graus) significativamente maior que o grupo controle na amostra total e nas subamostras estratificadas por gênero. Identificou-se como o melhor ponto de corte (cutoff) para o primeiro grupo uma inclinação tibial posterior $\geq 8^{\circ}$, atingindo uma sensibilidade de $63,3 \%$ e uma especificidade de $62,5 \%$. O primeiro grupo também apresentou proporção de slope tibial $\geq 8^{\circ}(63,3 \%)$, significativamente maior que o grupo controle $(37,5 \%)$, com razão de chances de 2,8 .

Conclusão Concluiu-se que o aumento da inclinação tibial posterior está associado com um maior risco para lesão do LCA por trauma indireto, principalmente para valores $\geq 8^{\circ}$,
DOI https://doi.org/

10.1055/s-0040-1712495. ISSN 0102-3616. (c) 2020. Sociedade Brasileira de Ortopedia e Traumatologia. All rights reserved.

This is an open access article published by Thieme under the terms of the Creative Commons Attribution-NonDerivative-NonCommercial-License, permitting copying and reproduction so long as the original work is given appropriate credit. Contents may not be used for commercial purposes, or adapted, remixed, transformed or built upon. (https://creativecommons.org/ licenses/by-nc-nd/4.0/)

Thieme Revinter Publicações Ltda., Rua do Matoso 170, Rio de Janeiro, RJ, CEP 20270-135, Brazil 


\author{
Keywords \\ - anterior cruciate \\ ligament \\ - ligaments \\ - knee injuries \\ - tibia
}

Methods Retrospective study, performed by analysis of medical records and digital radiographs of patients, present in a database of a tertiary orthopedic hospital. The sample consisted of two groups, the first group consisting of patients diagnosed with $\mathrm{ACL}$ injury by indirect trauma, and a control group matched by age.

Results Each group consisted of 275 patients, whose measurements of posterior tibial inclination were measured by three specialists. It was observed that the group of patients with $\mathrm{ACL}$ lesion presented a significantly higher tibial slope (in degrees) than the control group in the total sample and in the subsamples stratified by gender. The best cutoff point for the first group was identified as a posterior tibial inclination $\geq 8^{\circ}$, achieving a sensitivity of $63.3 \%$ and a specificity of $62.5 \%$. The first group also had a tibial slope ratio $\geq 8^{\circ}(63.3 \%)$, significantly higher than the control group (37.5\%), with an odds ratio of 2.8 .

Conclusion It was concluded that the increase of the posterior tibial inclination is associated with an increased risk for injury of the ACL by indirect trauma, mainly for values $\geq 8^{\circ}$.

\section{Introdução}

O ligamento cruzado anterior (LCA) é o principal restritor da translação anterior da tíbia sobre o fêmur, sendo responsável por $85 \%$ da estabilização anterior do joelho. ${ }^{1,2}$ Ele também atua limitando a rotação interna e restringindo secundariamente os estresses em valgo e varo. ${ }^{2-4}$

A lesão do LCA é uma das mais comuns lesões ligamentares do joelho, com crescente incidência devido ao número cada vez maior de indivíduos envolvidos com a prática de atividades esportivas. $^{2,4}$ Ocorre, predominantemente, secundária a um trauma indireto, com uma associação entre estresse em valgo do joelho e rotação interna da tíbia. ${ }^{2,4,5} \mathrm{O}$ não tratamento adequado de uma instabilidade anterior pode levar a lesões de outras estruturas ou alterações degenerativas em longo prazo. O seu tratamento cirúrgico tem bons resultados, apesar de nem sempre o paciente conseguir retornar às atividades esportivas com o mesmo desempenho de antes da lesão. ${ }^{5-7}$

A identificação de fatores de risco para a lesão do LCA durante a realização de atividades físicas e esportivas tornou-se um foco de pesquisa osteomuscular. A compreensão dos mecanismos que produzem esta instabilidade permite a identificação de pessoas com risco acrescido para que intervenções preventivas possam ser aplicadas. ${ }^{6,7}$

A inclinação posterior da tíbia tem sido cada vez mais estudada como um fator de risco potencial à lesão do LCA, mostrando resultados bastante variados entre seu aumento e a lesão ligamentar. ${ }^{6,8,9}$

Alguns estudos biomecânicos da articulação do joelho verificam que, durante uma carga de compressão axial, a inclinação posterior da tíbia age produzindo um componente de força que leva à anteriorização da tíbia em relação ao fêmur. ${ }^{8-12}$ Sabe-se que o LCA é o sistema de retenção primária contra este tipo de movimento do joelho, ou seja, um aumento da inclinação tibial posterior irá gerar um aumento do estresse neste ligamento. ${ }^{1,11,12}$ Apesar de alguns estudos sugerirem a relação da inclinação posterior do planalto tibial com a lesão do LCA, o nível de risco apresentado por este fator intrínseco permanece obscuro. $6,8,9$

A redução na ocorrência de lesões no LCA em indivíduos ativos jovens continua a ser um objetivo importante em medicina esportiva. O objetivo do presente estudo é avaliar, na população brasileira, a relação entre pacientes com lesão do LCA por trauma indireto e o aumento da inclinação tibial posterior.

\section{Material e Métodos}

Trata-se de um estudo retrospectivo, realizado através da análise de prontuários e de radiografias digitais de pacientes presentes em banco de dados de um hospital terciário de ortopedia e traumatologia no Brasil, no período de janeiro de 2014 a janeiro de 2016.

A amostra foi composta por dois grupos, sendo o Grupo I formado por pacientes com diagnóstico de lesão do LCA por trauma indireto. Durante o período do estudo, foram identificados 643 pacientes com lesão do LCA. Para formar o grupo I, foram excluídos os pacientes que não tinham prontuário evidenciando de forma clara o mecanismo de trauma como indireto. Outros critérios de exclusão foram radiografias do joelho que inviabilizavam a mensuração confiável da inclinação tibial posterior (qualidade ruim, alterações radiológicas por cirurgias prévias ou osteoartrose).

Um grupo controle (Grupo II) foi formado a partir de um banco de dados de radiografias do joelho, pareado por idade com o Grupo I. Foi excluído qualquer paciente com evolução do prontuário evidenciando lesão ligamentar do joelho. Também foram excluídos os sujeitos de pesquisa com imagem que impossibilitava a mensuração confiável do slope tibial, como descrito para o grupo I. Após análise dos critérios de exclusão, cada grupo foi composto por 275 pacientes. A idade da amostra variou de 16 a 55 anos. A - Tabela 1 fornece dados relativos à idade e à distribuição quanto ao gênero. 
Tabela 1 Caracterização da amostra quanto à idade e quanto ao gênero

\begin{tabular}{|c|c|c|c|}
\hline Variável & $\begin{array}{l}\text { Lesão do } \\
\text { LCA por } \\
\text { Trauma } \\
\text { indireto } \\
(n=275)\end{array}$ & $\begin{array}{l}\text { Grupo } \\
\text { controle } \\
(n=275)\end{array}$ & $\begin{array}{l}\text { Total } \\
(n=550)\end{array}$ \\
\hline \multicolumn{4}{|l|}{ Idade (anos) } \\
\hline Média (DP) & $33,0(8,8)$ & $38,4(9,7)$ & $35,7(9,7)$ \\
\hline \multicolumn{4}{|l|}{ Gênero (N) } \\
\hline Masculino & $241(87,6 \%)$ & $212(77,1 \%)$ & $453(82,4 \%)$ \\
\hline Feminino & $34(12,4 \%)$ & $63(22,9 \%)$ & $97(17,6 \%)$ \\
\hline
\end{tabular}

Abreviação: DP, desvio padrão; LCA, ligamento cruzado anterior.

Todos os pacientes foram submetidos a estudo radiográfico de acordo com a rotina preconizada pela instituição. Foi usado o aparelho de raios-X Shimatzu (RADspeed MF, Shimadzu, Kyoto, Japão) de $500 \mathrm{~mA}$ com técnica de $50 \mathrm{KV}$ e $25 \mathrm{~mA}$. Um filme de $30 \times 40 \mathrm{~cm}$ foi colocado a uma distância de um metro da ampola do aparelho radiográfico digital. Foram, então, obtidas as imagens em incidência lateral (perfil) em semiflexão de $30^{\circ}$.

Os pacientes tiveram suas radiografias em perfil do joelho analisadas, e sua inclinação tibial posterior foi mensurada por três especialistas em ortopedia que desconheciam a qual grupo cada um dos pacientes pertencia. Essa medida foi realizada traçando-se uma linha na cortical posterior da

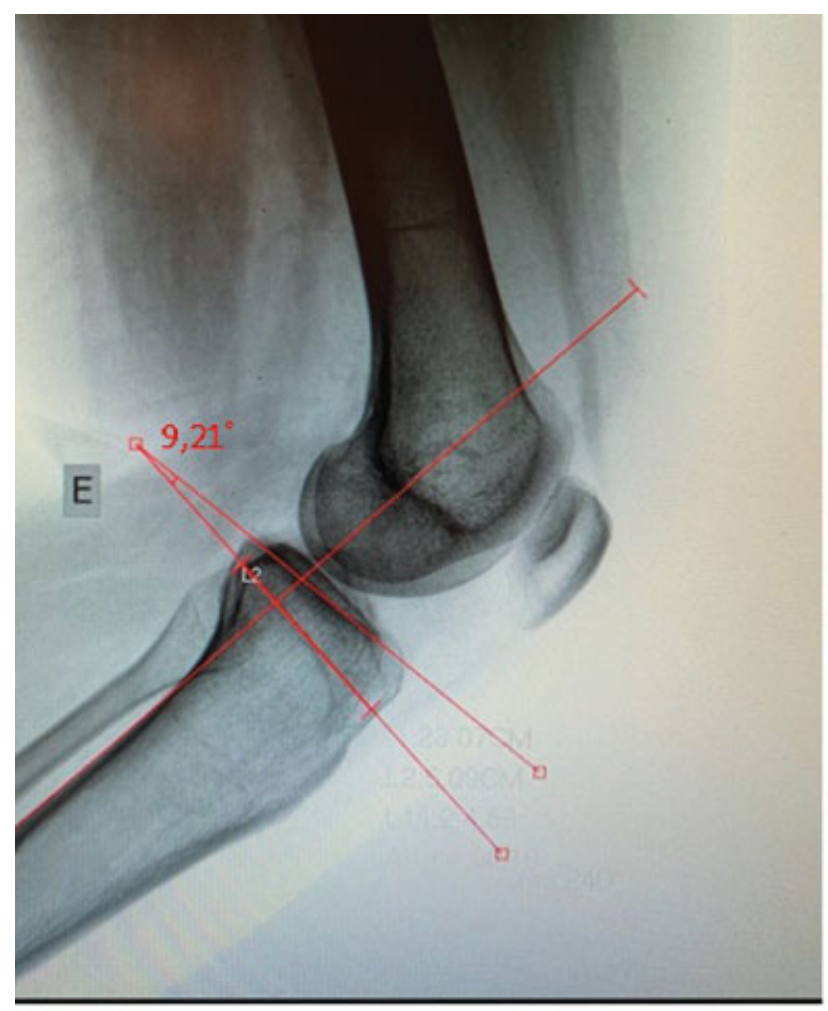

Fig. 1 Demonstração de mensuração da inclinação tibial posterior. tíbia e outra na superfície articular proximal da tíbia. 0 ângulo formado entre a perpendicular à linha da cortical posterior e a linha da superfície articular correspondeu à medida da inclinação tibial posterior, conforme descrito na - Figura 1 e determinado pela técnica de Hohmann et al. ${ }^{13}$

A análise estatística foi composta pelo teste $t$ de Student para amostras independentes na comparação de dados contínuos entre o grupo com lesão do LCA por mecanismo indireto e o grupo controle, e pelo teste de qui-quadrado (X2) na comparação de dados categóricos. Na associação entre variáveis contínuas, foi utilizado o coeficiente de correlação de Pearson.

A curva Receiver Operating Characteristic (ROC) foi construída para identificar o melhor ponto de corte da inclinação tibial posterior para trauma indireto. A força da associação entre a inclinação tibial posterior elevada com o trauma indireto foi medida pela razão de chances (odds ratio [OR]) e seu respectivo intervalo de confiança (IC) de $95 \%$.

A normalidade na distribuição dos dados foi avaliada pelo teste de Kolmogorov-Smirnov e análise gráfica do histograma. $\mathrm{O}$ critério de determinação de significância adotado foi o nível de 5\%. A análise estatística foi processada pelo software IBM SPSS Statistics for Windows, versão 20.0 (IBM Corp., Armonk, NY, EUA). O estudo foi previamente aprovado pelo comitê de ética em pesquisa do hospital em que o estudo foi realizado sob o número CAAE 79853617.0.0000.5273.

\section{Resultados}

Os valores da inclinação tibial posterior variaram de $2,6^{\circ}$ a $18,1^{\circ}$ no primeiro grupo, com média de $9,1^{\circ}$, e de 0 a $17,6^{\circ}$ no segundo, com valor médio de $7,3^{\circ}$. Avaliando-se as variáveis inclinação tibial posterior e os gêneros como um todo, segundo o teste $t$ de Student, verificamos não haver associação significativa ( $p=0,66$ ), ou seja, os homens não apresentaram slope tibial médio ( $8,2 \pm 2,9$ graus) significativamente diferente das mulheres $(8,1 \pm 2,8$ graus).

Tabela 2 Slope tibial (em graus) de acordo com os grupos e estratificado por gênero. Teste $t$ de Student para amostras independentes

\begin{tabular}{|c|c|c|c|}
\hline Amostra & $\begin{array}{l}\text { Lesão do } \\
\text { LCA por } \\
\text { Trauma } \\
\text { indireto }\end{array}$ & $\begin{array}{l}\text { Grupo } \\
\text { Controle }\end{array}$ & valor-p \\
\hline \multicolumn{4}{|c|}{ Todos $(n=275 \times 275)$} \\
\hline Média (DP) & $9,1(2,9)$ & $7,3(2,6)$ & \multirow[t]{2}{*}{$<0,0001$} \\
\hline Mínimo-máximo & $2,6-18,1$ & $0-17,6$ & \\
\hline \multicolumn{4}{|c|}{ Homens $(n=241 \times 212)$} \\
\hline Média (DP) & $9,0(2,9)$ & $7,3(2,6)$ & \multirow[t]{2}{*}{$<0,0001$} \\
\hline Mínimo-máximo & $2,6-18,1$ & $0,10-17,6$ & \\
\hline \multicolumn{4}{|c|}{ Mulheres $(n=34 \times 63)$} \\
\hline Média (DP) & $9,3(3,0)$ & $7,4(2,5)$ & \multirow[t]{2}{*}{0,001} \\
\hline Mínimo-máximo & $3,1-14,6$ & $0-12,5$ & \\
\hline
\end{tabular}

Abreviações: DP, desvio padrão; LCA, ligamento cruzado anterior. 


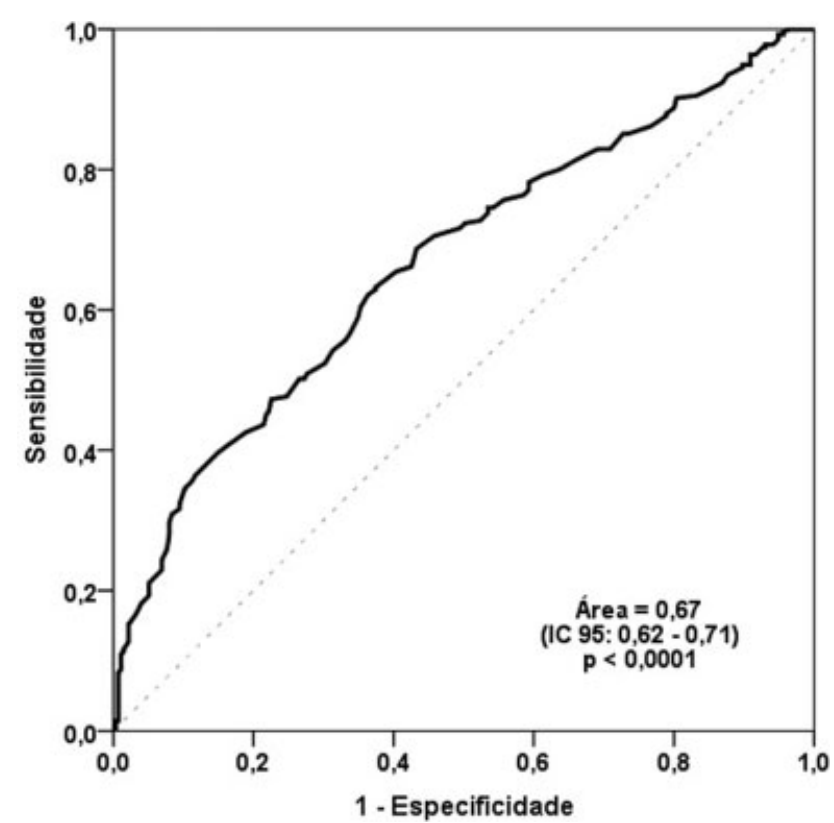

Fig. 2 Curva ROC do slope tibial (em graus) para os pacientes com lesão do LCA por trauma indireto.

Ao realizarmos a associação entre slope tibial e os dois grupos em estudo, observamos que o grupo dos pacientes com lesão do LCA por trauma indireto apresentou slope tibial (em graus) significativamente maior que o grupo controle na amostra total e nas subamostras estratificadas por gênero. A - Tabela 2 fornece a descritiva do slope tibial (média, desvio padrão [DP], mínimo e máximo, em graus) de acordo com os grupos e o correspondente nível descritivo (valor-p) do teste $t$ de Student para amostras independentes, no total da amostra e estratificado por gênero (homens e mulheres).

A - Figura 2 ilustra a curva ROC da inclinação tibial posterior para o grupo com lesão do LCA por trauma indireto na amostra total. A acurácia global de um teste pode ser descrita como a área sob a curva ROC, quanto maior a área, ou seja, mais próximo de 1 , melhor é o teste.

Observou-se uma área de 0,67 com IC95\% de 0,62 a 0,71, expressando um poder discriminatório "moderado/regular," com valor significativo $(p<0,0001)$. Além disso, considerando o grupo controle como categoria de referência, podese identificar o melhor ponto de corte (cutoff) para o primeiro grupo, que, segundo a curva ROC nesta amostra em estudo, foi uma inclinação tibial posterior $\geq 8^{\circ}$, atingindo uma sensibilidade de $63,3 \%$ e uma especificidade de $62,5 \%$.

A - Tabela 3 fornece a frequência (n) e o percentual (\%) do slope tibial $\geq 8^{\circ}$, segundo os grupos em análise, o correspondente nível descritivo (valor-p) e a razão de chances (RC) para lesão do LCA por trauma indireto com o respectivo IC95\%, na amostra total. Observou-se, na amostra total, que o grupo com lesão do LCA por trauma indireto apresentou proporção de slope tibial $\geq 8^{\circ}(63,3 \%)$ significativamente maior que 0 grupo controle (37,5\%), com razão de chances de 2,8 (IC 95\%: 2,04-4,07) (-Figura 3).

Já na -Tabela 4, observou-se que o grupo I apresentou proporção de inclinação tibial posterior $\geq 8^{\circ}$ significativamente maior que o grupo controle ao estratificar em subamostras de acordo com o gênero, com razão de chances $\sim 3$ para lesão do LCA por trauma indireto.

\section{Discussão}

A associação entre lesão do LCA e a inclinação tibial posterior está bem documentada na literatura mesmo que ainda não exista, em caráter bem sedimentado, o nível de risco que tal associação possa ter. 0 presente estudo procurou avaliar, especificamente, a importância do grau de inclinação tibial posterior em pacientes com lesão do LCA originadas a partir de trauma indireto. Frente aos resultados encontrados, percebe-se que não há uma associação entre o gênero do paciente e a intensidade da inclinação tibial posterior, diferentemente do que foi encontrado por Hohmann et al., ${ }^{13}$ que encontrou angulações maiores no sexo feminino. Diante de condições iguais de exposição, sabe-se que o sexo feminino tem um maior risco de lesão do LCA que o sexo masculino; ;,14 entretanto, a inclinação tibial posterior não poderia ser considerada, segundo resultados encontrados, umas das razões para esse risco aumentado.

A relação entre a inclinação tibial posterior e pacientes com lesão do LCA por trauma indireto mostrou que um aumento da angulação representaria um risco aumentado à estrutura do LCA quando comparado a um grupo controle, comprovando uma interferência importante da anatomia e da biomecânica do joelho na estabilidade da articulação.

Essa relação já foi anteriormente descrita por alguns autores $^{9,15-18}$ que demonstraram que a inclinação tibial posterior tem um efeito adverso na cinemática do joelho. Em um modelo de cadáver, Dejour et al. ${ }^{15}$ mostraram um aumento de $6 \mathrm{~mm}$ na translação anterior da tíbia para cada aumento de $10^{\circ}$ na inclinação tibial posterior. Da mesma forma, Giffin et al. ${ }^{16}$ demonstraram um aumento significativo na translação anterior da tíbia se a inclinação posterior fosse aumentada em $4,4^{\circ}$ após uma osteotomia tibial alta na cunha de abertura. Fening et al. ${ }^{9}$ realizaram osteotomias tibiais altas na cunha de abertura e também relataram um aumento na translação anterior da tíbia com um aumento na inclinação tibial.

McLean et al. ${ }^{19}$ sugeriram que a compressão axial de um joelho com uma maior inclinação do planalto tibial lateral,

Tabela 3 Distribuição dos pacientes com inclinação tibial posterior $\geq 8^{\circ}$ segundo os grupos

\begin{tabular}{|l|l|l|l|l|l|}
\hline Slope tibial & Lesão do LCA por trauma indireto & Grupo Controle & valor-p & RC & IC95\% \\
\hline$\geq 8^{\circ}$ & $174(63,3 \%)$ & $103(37,5 \%)$ & $<0,0001$ & 2,87 & $2,04-4,07$ \\
\cline { 1 - 3 } & $101(36,7 \%)$ & $172(62,5 \%)$ & & & \\
\hline
\end{tabular}

Abreviações: IC, intervalo de confiança; LCA, ligamento cruzado anterior; RC, razão de chances.

Teste de $x^{2}$. 


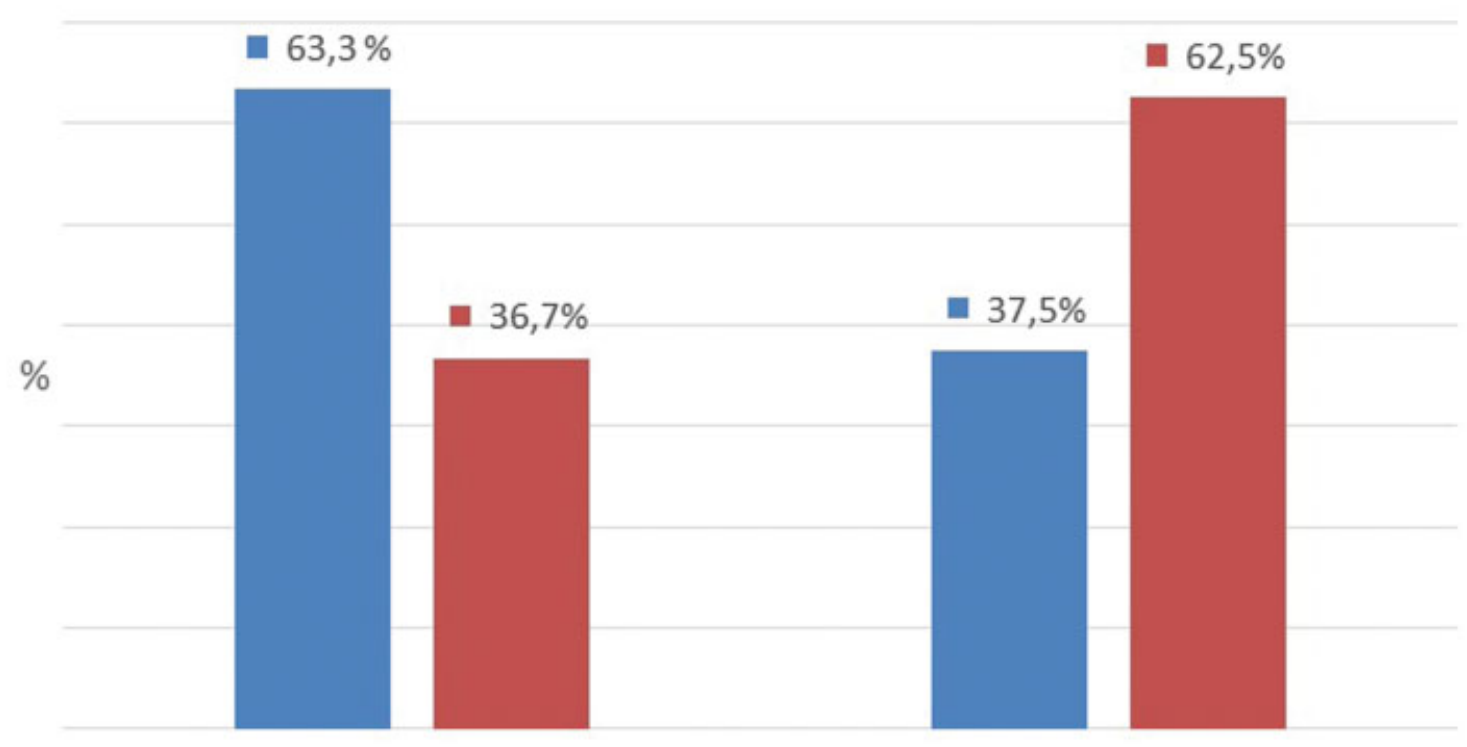

Slope $\geq 8^{\circ} \quad$ Slope $<8^{\circ}$

Fig. 3 Slope tibial $\geq 8^{\circ}$ segundo os grupos em estudo.

Tabela 4 Slope tibial $\geq 8$ graus segundo o tipo de trauma na amostra total e estratificada por gênero e faixa etária

\begin{tabular}{|c|c|c|c|c|c|}
\hline Slope tibial & Lesão do LCA por trauma indireto & Grupo controle & valor-p & RC & IC $95 \%$ \\
\hline \multicolumn{6}{|c|}{ Todos $(n=275 \times 275)$} \\
\hline$\geq 8$ graus & $174(63,3 \%)$ & $103(37,5 \%)$ & \multirow[t]{2}{*}{$<0,0001$} & \multirow[t]{2}{*}{2,87} & \multirow[t]{2}{*}{$2,04-4,07$} \\
\hline$<8$ graus & $101(36,7 \%)$ & $172(62,5 \%)$ & & & \\
\hline \multicolumn{6}{|c|}{ Homens $(n=241 \times 212)$} \\
\hline$\geq 8$ graus & $149(61,8 \%)$ & $74(34,9 \%)$ & \multirow[t]{2}{*}{0,011} & \multirow[t]{2}{*}{3,02} & \multirow[t]{2}{*}{$2,05-4,43$} \\
\hline$<8$ graus & $92(38,2 \%)$ & $138(65,1 \%)$ & & & \\
\hline \multicolumn{6}{|c|}{ Mulheres $(n=34 \times 63)$} \\
\hline$\geq 8$ graus & $25(73,5 \%)$ & $29(46,0 \%)$ & \multirow[t]{2}{*}{$<0,0001$} & \multirow[t]{2}{*}{3,25} & \multirow[t]{2}{*}{$1,31-8,08$} \\
\hline$<8$ graus & $9(26,5 \%)$ & $34(54,0 \%)$ & & & \\
\hline
\end{tabular}

Abreviações: IC, intervalo de confiança; LCA, ligamento cruzado anterior; RC, razão de chances.

Teste de $x^{2}$.

em comparação com a do planalto tibial medial, pode causar um maior movimento anterior do compartimento lateral da tíbia, em comparação com o outro, gerando um estresse em rotação interna da tíbia em relação ao fêmur, aumentando ainda mais a carga no LCA.

A análise estatística do presente estudo encontrou que pacientes com uma angulação $\geq 8^{\circ}$ possuem 3 vezes mais chances de lesionar o LCA através de um trauma indireto que pacientes com angulação $<8^{\circ}$, independente do gênero.

Alguns autores defendem a realização da osteotomia deflexora como tratamento cirúrgico para pacientes com inclinação tibial posterior excessiva associada à ruptura do LCA. $^{20,21}$ Dejour et al. ${ }^{20}$ avaliaram, retrospectivamente, uma série de pacientes com slope tibial $>12^{\circ}$ e que foram sub- metidos a uma segunda revisão de reconstrução do LCA associada a osteotomia deflexora. Após um seguimento mínimo de 2 anos, os 9 pacientes do estudo, que atenderam aos critérios adotados, estavam livres de complicações e com escores funcionais satisfatórios, justificando o procedimento para casos selecionados.

O presente estudo apresentou limitações por ser retrospectivo. Isso levou à exclusão de sujeitos de pesquisa, devido às informações incompletas nos prontuários, além de dificultar o pareamento dos grupos quanto a outros critérios importantes, como o nível de atividade esportiva desempenhada ou quanto a outros fatores de risco associados para lesão do LCA, como deformidades angulares. 
52 Análise da inclinação tibial posterior como fator de risco para lesão do LCA Sousa Filho et al.

\section{Conclusão}

Conclui-se que o aumento da inclinação tibial posterior está associado com um maior risco para lesão do LCA por trauma indireto, independente do gênero. Dessa forma, medidas corretivas devem ser consideradas, principalmente, para aqueles que apresentarem inclinação tibial excessiva associada à instabilidade anterior do joelho.

Conflito de Interesses

Os autores declaram não haver conflito de interesses.

\section{Referências}

1 Butler DL, Noyes FR, Grood ES. Ligamentous restraints to anteriorposterior drawer in the human knee. A biomechanical study. J Bone Joint Surg Am 1980;62(02):259-270

2 Dargel J, Gotter M, Mader K, Pennig D, Koebke J, Schmidt-Wiethoff R. Biomechanics of the anterior cruciate ligament and implications for surgical reconstruction. Strateg Trauma Limb Reconstr 2007;2(01):1-12

3 Dennis DA, Mahfouz MR, Komistek RD, Hoff W. In vivo determination of normal and anterior cruciate ligament-deficient knee kinematics. J Biomech 2005;38(02):241-253

4 Logan M, Dunstan E, Robinson J, Williams A, Gedroyc W, Freeman M. Tibiofemoral kinematics of the anterior cruciate ligament (ACL)-deficient weightbearing, living knee employing vertical access open "interventional" multiple resonance imaging. Am J Sports Med 2004;32(03):720-726

5 Domnick C, Raschke MJ, Herbort M. Biomechanics of the anterior cruciate ligament: Physiology, rupture and reconstruction techniques. World J Orthop 2016;7(02):82-93

6 Wordeman SC, Quatman CE, Kaeding CC, Hewett TE. In vivo evidence for tibial plateau slope as a risk factor for anterior cruciate ligament injury: a systematic review and meta-analysis. Am J Sports Med 2012;40(07):1673-1681

7 Smith HC, Vacek P, Johnson RJ, et al. Risk factors for anterior cruciate ligament injury: a review of the literature - part 1: neuromuscular and anatomic risk. Sports Health 2012;4(01):69-78

8 Zeng C, Cheng L, Wei J, et al. The influence of the tibial plateau slopes on injury of the anterior cruciate ligament: a meta-analysis. Knee Surg Sports Traumatol Arthrosc 2014;22(01):53-65
9 Fening SD, Kovacic J, Kambic H, McLean S, Scott J, Miniaci A. The effects of modified posterior tibial slope on anterior cruciate ligament strain and knee kinematics: a human cadaveric study. J Knee Surg 2008;21(03):205-211

10 Dejour H, Bonnin M. Tibial translation after anterior cruciate ligament rupture. Two radiological tests compared. J Bone Joint Surg Br 1994;76(05):745-749

11 Torzilli PA, Deng X, Warren RF. The effect of joint-compressive load and quadriceps muscle force on knee motion in the intact and anterior cruciate ligament-sectioned knee. Am J Sports Med 1994;22(01):105-112

12 Voos JE, Suero EM, Citak M, et al. Effect of tibial slope on the stability of the anterior cruciate ligament-deficient knee. Knee Surg Sports Traumatol Arthrosc 2012;20(08):1626-1631

13 Hohmann E, Bryant A, Reaburn P, Tetsworth K. Is there a correlation between posterior tibial slope and non-contact anterior cruciate ligament injuries? Knee Surg Sports Traumatol Arthrosc 2011;19(Suppl 1):S109-S114

14 Alentorn-Geli E, Mendiguchía J, Samuelsson K, et al. Prevention of anterior cruciate ligament injuries in sports. Part I: systematic review of risk factors in male athletes. Knee Surg Sports Traumatol Arthrosc 2014;22(01):3-15

15 Dejour H, Walch G, Chambat P, Ranger P. Active sub-luxation in extension: a new concept of study of the ACL-deficient knee. Am J Knee Surg 1988;1:204-211

16 Giffin JR, Vogrin TM, Zantop T, Woo SL, Harner CD. Effects of increasing tibial slope on the biomechanics of the knee. Am J Sports Med 2004;32(02):376-382

17 Hohmann E, Bryant AL. Closing or opening wedge high tibial osteotomy: watch out for the slope. Oper Tech Orthop 2007;17(01):17-38

18 Liu W, Maitland ME. Influence of anthropometric and mechanical variations on functional instability in the ACL-deficient knee. Ann Biomed Eng 2003;31(10):1153-1161

19 McLean SG, Lucey SM, Rohrer S, Brandon C. Knee joint anatomy predicts high-risk in vivo dynamic landing knee biomechanics. Clin Biomech (Bristol, Avon) 2010;25(08):781-788

20 Dejour D, Saffarini M, Demey G, Baverel L. Tibial slope correction combined with second revision ACL produces good knee stability and prevents graft rupture. Knee Surg Sports Traumatol Arthrosc 2015;23(10):2846-2852

21 Sonnery-Cottet B, Archbold P, Cucurulo T, et al. The influence of the tibial slope and the size of the intercondylar notch on rupture of the anterior cruciate ligament. J Bone Joint Surg Br 2011;93 (11):1475-1478 